PROCEEDINGS OF THE

AMERICAN MATHEMATICAL SOCIETY

Volume 129, Number 3, Pages 731-738

S 0002-9939(00)05800-7

Article electronically published on August 30, 2000

\title{
ALMOST PERIODIC HYPERFUNCTIONS
}

\author{
JAEYOUNG CHUNG, SOON-YEONG CHUNG, DOHAN KIM, AND HEE JUNG KIM
}

(Communicated by Jonathan M. Borwein)

\begin{abstract}
We characterize the almost periodic hyperfunctions by showing that the following statements are equivalent for any bounded hyperfunction $T$. (i) $T$ is almost periodic. (ii) $T * \varphi \in C_{a p}$ for every $\varphi \in \mathcal{F}$. (iii) There are two functions $f, g \in C_{a p}$ and an infinite order differential operator $P$ such that $T=P\left(D^{2}\right) f+g$. (iv) The Gauss transform $u(x, t)=T * E(x, t)$ of $T$ is almost periodic for every $t>0$. Here $C_{a p}$ is the space of almost periodic continuous functions, $\mathcal{F}$ is the Sato space of test functions for the Fourier hyperfunctions, and $E(x, t)$ is the heat kernel. This generalizes the result of Schwartz on almost periodic distributions and that of Cioranescu on almost periodic (non-quasianalytic) ultradistributions to the case of hyperfunctions.
\end{abstract}

\section{INTRODUCTION}

Let $f(x)$ be a complex valued continuous function defined on $\mathbb{R}$. A number $\tau$ is called an $\epsilon$-almost period of $f(x)$ if $\sup _{-\infty<x<\infty}|f(x+\tau)-f(x)| \leq \epsilon$. If for any $\epsilon>0$ there exists a number $l(\epsilon)$ such that every interval of length $l(\epsilon)$ contains an $\epsilon$-almost period of $f$, then $f(x)$ is said to be almost periodic.

It is well known that the following three statements are equivalent:

(i) $f$ is an almost periodic function.

(ii) The set of translations $f_{h}$ for $h \in \mathbb{R}$ forms a relatively compact set with respect to the uniform topology.

(iii) $f(x)$ is the uniform limit of a sequence of (generalized) trigonometric polynomials

$$
P_{m}(x)=\sum_{n=1}^{l} \alpha_{n} \exp i \lambda_{n} x, \quad \lambda_{n} \in \mathbb{R} .
$$

The definition of almost periodicity for the continuous functions cannot carry over to generalized functions. Instead, the equivalent statements (ii) or (iii) can be used to define almost periodic generalized functions.

Schwartz [S] used (ii) to define almost periodic distributions in the sense of Stepanoff and showed that the following statements are equivalent for any bounded

Received by the editors May 4, 1999.

1991 Mathematics Subject Classification. Primary 46F15, 35K05, $42 \mathrm{~B} 05$.

Key words and phrases. Almost periodic, hyperfunction, ultradistribution.

The first and second authors were partially supported by KOSEF (1999-2-101-001-5). The third and fourth authors were partially supported by BK21.

(C)2000 American Mathematical Society 
distribution $T$ :

(1) $T$ is almost periodic.

(2) $T$ is the finite sum of derivatives of functions in $C_{a p}$.

(3) $T * \varphi \in C_{a p}$ for all $\varphi \in \mathcal{D}$.

Here, $C_{a p}$ is the space of almost periodic functions.

Cioranescu [C2] used (iii) instead to define almost periodic non-quasianalytic ultradistributions of Beurling type and showed that the following statements are equivalent for any bounded ultradistribution $T$ :

(1) $T$ is almost periodic.

(2) $T * \varphi \in C_{a p}$ for every $\varphi \in \mathcal{D}^{\left(M_{p}\right)}$ which is the space of ultradifferentiable functions of class $\left(M_{p}\right)$ in $[\mathrm{K}]$.

(3) There are two functions $f, g \in C_{a p}$ and an ultradifferential operator $P$ of class $\left(M_{p}\right)$ such that $T=P\left(D^{2}\right) f+g$.

In this paper we generalize the above results of Schwartz and Cioranescu to the case of hyperfunctions and show that the following statements are equivalent for any bounded hyperfunction $T$ :

(1) $T$ is almost periodic.

(2) $T * \varphi \in C_{a p}$ for every $\varphi \in \mathcal{F}$ which is the Sato space defined in Section 2 .

(3) There exist two functions $f$ and $g$ belonging to $C_{a p}$ and an ultradifferential operator $P$ of class $\left\{p !^{2}\right\}$ such that $T=P\left(D^{2}\right) f+g$.

(4) The Gauss transform $u(x, t)=(T * E)(x, t)$ of $T$ is almost periodic for each $t>0$, where $E(x, t)$ is the heat kernel.

We also obtain the similar result for quasianalytic ultradistributions $T$, which, in fact, includes all the above results of Schwartz, Cioranescu and ourselves. Although Cioranescu imposed the condition (M.3) in addition to (M.1) and (M.2) we impose a much weaker condition $(\mathrm{C})$ to prove our characterization theorem for quasi-analytic ultradistributions.

For the proof we apply the characterization of bounded hyperfunctions in ChungKim-Lee [CKL] and make use of the heat kernel method which represents various generalized functions as the initial values of solutions of the heat equation with suitable growth conditions.

\section{Preliminaries}

Let $M_{p}, p=0,1,2, \cdots$, be a sequence of positive numbers. We impose the following conditions on $M_{p}$ :

(M.1) $M_{p}^{2} \leq M_{p-1} M_{p+1}, \quad p \in \mathbb{N}$;

(M.2) There exist positive constants $A$ and $H$ such that

$$
M_{p} \leq A H^{p} \min _{0 \leq q \leq p} M_{q} M_{p-q}, \quad p \in \mathbb{N}_{0} .
$$

The following strong non-quasianalyticity condition (M.3) has been assumed in the main result of Cioranescu [C2], but we do not impose the condition (M.3) to study hyperfunctions and a class of quasianalytic ultradistributions.

(M.3) There exists a positive constant $A$ such that

$$
\sum_{q=p+1}^{\infty} M_{q-1} / M_{q} \leq A p M_{p} / M_{p+1}, \quad p \in \mathbb{N} .
$$


We briefly give definitions of Fourier hyperfunctions and bounded hyperfunctions. We refer to $\mathrm{KCK}, \mathrm{CKL}, \mathrm{C} 2$ for more details. We denote by $\mathcal{F}$ the Sato space of all infinitely differentiable functions $\varphi$ in $\mathbb{R}$ satisfying

$$
\|\varphi\|_{h, k}=\sup _{\substack{x \in \mathbb{R} \\ p \in \mathbb{N}_{0}}} \frac{\left|\varphi^{(p)}(x)\right| \exp k|x|}{h^{|p|} p !}<\infty
$$

for some $h, k>0$. Also, we denote by $\mathcal{F}^{\prime}$ the strong dual of $\mathcal{F}$ and call its elements Fourier hyperfunctions.

We denote by $\mathcal{D}_{L^{1}}^{\left\{M_{p}\right\}}$ the space of functions $\varphi \in C^{\infty}(\mathbb{R})$ satisfying

$$
\|\varphi\|_{L^{1}, h}=\sup _{p} \frac{\left\|\varphi^{(p)}\right\|_{L^{1}}}{h^{p} M_{p}}<\infty
$$

for some constant $h>0$. We say that $\varphi_{j} \rightarrow 0$ as $j \rightarrow \infty$ in $\mathcal{D}_{L^{1}}^{\left\{M_{p}\right\}}$ if $\left\|\varphi_{j}\right\|_{L^{1}, h} \rightarrow 0$ as $j \rightarrow \infty$ for some $h>0$. Also, we denote by $\mathcal{D}_{L^{\infty}}^{\left\{M_{p}\right\}}$ the strong dual space of $\mathcal{D}_{L^{1}}^{\left\{M_{p}\right\}}$ and call its elements bounded ultradistributions of Roumieu type. In particular, we denote the space $\mathcal{D}_{L^{1}}^{\{p !\}}$ by $\mathcal{A}_{L^{1}}$ and denote by $\mathcal{B}_{L^{\infty}}$ the dual space of $\mathcal{A}_{L^{1}}$ and call its elements bounded hyperfunctions. It is easy to see the following topological inclusions: $\mathcal{F} \hookrightarrow \mathcal{A}_{L^{1}}, \mathcal{B}_{L^{\infty}} \hookrightarrow \mathcal{F}^{\prime}$.

Let $D=-i d / d t$. Then an operator of the form $P(D)=\sum a_{n} D^{n}$ is called an ultradifferential operator (of class $\left\{M_{p}\right\}$ ) if for every $L$ there exists $C$ such that

$$
\left|a_{n}\right| \leq C L^{n} / M_{n}, n \in \mathbb{N}_{0} .
$$

The following result establishes the existence of a parametrix of an ultradifferential operator, which will be very useful later.

Lemma $2.1(\underline{\mathrm{M}}])$. For any $h>0$ and $\epsilon>0$ there exist functions $v(t) \in C_{c}^{\infty}([0, \epsilon])$, $w(t) \in C_{c}^{\infty}([\epsilon / 2, \epsilon])$ and an ultradifferential operator $P(d / d t)$ such that

$$
\begin{gathered}
\left|v^{(k)}(t)\right| \leq C h^{-k} k !^{2}, \quad k=0,1, \cdots, \\
|v(t)| \leq C \exp (-h / t), \quad 0<t<\infty, \\
P(d / d t)=\sum_{k=0}^{\infty} a_{k}(d / d t)^{k},\left|a_{k}\right| \leq C_{1} h_{1}^{k} / k !^{2}, \quad 0<h_{1}<h, \\
P(d / d t) v(t)+w(t)=\delta .
\end{gathered}
$$

\section{Almost periodic hyperfunctions}

We first recall the characterization of the bounded hyperfunctions as in CKL]. Let $E(x, t)$ be the heat kernel

$$
E(x, t)= \begin{cases}\frac{1}{\sqrt{4 \pi t}} \exp \left(-x^{2} / 4 t\right), & t>0, \\ 0, & t \leq 0 .\end{cases}
$$

Note that $E(x, t)$ belongs to the Sato space $\mathcal{F}$ for each $t>0$ and $E(x-\cdot, t)$ belongs to $\mathcal{A}_{L^{1}}$ for each $(x, t) \in \mathbb{R} \times \mathbb{R}^{+}$. So, for each $T \in \mathcal{B}_{L^{\infty}}$ its Gauss transform $u(x, t)=T_{y}\left(E(x-y, t)\right.$ is a $C^{\infty}$ function in $\mathbb{R} \times \mathbb{R}^{+}$. 
Theorem 3.1 ([CKL $]$ ). The following statements are equivalent:

(i) $T \in \mathcal{B}_{L^{\infty}}$.

(ii) $T * \varphi \in L^{\infty}$ for every $\varphi \in \mathcal{F}$.

(iii) There exist two functions $f$ and $g$ belonging to $C_{b}$ and an ultradifferential operator $P$ of class $\left\{p !^{2}\right\}$ such that $T=P\left(D^{2}\right) f+g$.

(iv) The Gauss transform $u(x, t)$ of $T$ belongs to $C^{\infty}\left(\mathbb{R}_{+}^{2}\right)$ and satisfies the following:

$$
\left(\partial_{t}-\Delta\right) u(x, t)=0 \quad \text { in } \mathbb{R}_{+}^{2} ;
$$

for every $\epsilon>0$ there exists a constant $C>0$ such that

$$
\|u(x, t)\|_{L^{\infty}(\mathbb{R})} \leq C e^{\epsilon / t} \quad \text { in } \mathbb{R}_{+}^{2}
$$

and $u(\cdot, t) \rightarrow T$ as $t \rightarrow 0^{+}$in the sense that

$$
T(\varphi)=\lim _{t \rightarrow 0^{+}} \int_{\mathbb{R}} u(x, t) \varphi(x) d x, \quad \varphi \in \mathcal{A}_{L^{1}} .
$$

Here, $C_{b}$ is the space of bounded continuous functions on $\mathbb{R}$.

Using the equivalent condition (iii) in Section 1 we now define almost periodic hyperfunctions.

Definition 3.2. A hyperfunction $T \in \mathcal{B}_{L^{\infty}}$ is called almost periodic if $T$ is the limit of a sequence of trigonometric polynomials $P_{m}(x)=\sum_{n=1}^{k(m)} \alpha_{n} \exp \left(i \lambda_{n} x\right)$ in the space $\mathcal{B}_{L^{\infty}}$ with respect to the strong topology where $\lambda_{n} \in \mathbb{R}$ and $\alpha_{n} \in \mathbb{C}$ depend on $m$.

For the proof of our main result we need the following lemmas.

Lemma 3.3 ([CKL] $)$. For any $\varphi \in \mathcal{A}_{L^{1}}$, let

$$
\varphi_{t}(x)=\int E(x-y, t) \varphi(y) d y, \quad t>0 .
$$

Then $\varphi_{t} \in \mathcal{A}_{L^{1}}$ for every $t>0$ and $\varphi_{t} \rightarrow \varphi$ in $\mathcal{A}_{L^{1}}$ as $t \rightarrow 0^{+}$.

We now prove the continuity of the ultradifferential operator.

Lemma 3.4. Let $P(d / d t)$ be an ultradifferential operator of class $\left\{p !^{2}\right\}$. Then the operator $P\left(D^{2}\right): \mathcal{A}_{L^{1}} \longrightarrow \mathcal{A}_{L^{1}}$ is a continuous linear mapping.

Proof. Let $\varphi \in \mathcal{A}_{L^{1}}$. Then there exists $s>0$ such that $\left\|\varphi^{(p)}\right\|_{L^{1}} \leq s^{p} p !\|\varphi\|_{L^{1}, s}$ for all $p$. Using the inequalities (2.1) we obtain that for some constant $C$,

$$
\begin{aligned}
\left\|\left(a_{k}\left(D^{2}\right)^{k} \varphi(x)\right)^{(p)}\right\|_{L^{1}} & \leq\left|a_{k}\right|\left\|\varphi^{(2 k+p)}\right\|_{L^{1}} \\
& \leq\left|a_{k}\right| s^{(2 k+p)}(2 k+p) !\|\varphi\|_{L^{1}, s} \\
& \leq C\left(L 3^{2} s^{2}\right)^{k}(3 s)^{p} p !\|\varphi\|_{L^{1}, s},
\end{aligned}
$$

since $(2 k+p) ! \leq 3^{2 k+p}(k !)^{2} p$ !. Therefore we obtain

$$
\left\|a_{k}\left(D^{2}\right)^{k} \varphi\right\|_{L^{1}, 3 s} \leq C\left(L 3^{2} s^{2}\right)^{k}\|\varphi\|_{L^{1}, s} .
$$

If $L$ is so small that $L 3^{2} s^{2} \leq 1 / 2$, then $\left\|a_{k}\left(D^{2}\right)^{k} \varphi\right\|_{L^{1}, 3 s} \leq C(1 / 2)^{k}\|\varphi\|_{L^{1}, s}$. It follows easily that $\left\|P\left(D^{2}\right) \varphi\right\|_{L^{1}, 3 s} \leq 2 C\|\varphi\|_{L^{1}, s}$. Since the constant $C$ is independent of $\varphi$ we complete the proof. 
We are now in a position to state and prove the main result.

Theorem 3.5. For $T \in \mathcal{B}_{L^{\infty}}$ the following statements are equivalent:

(i) $T$ is almost periodic.

(ii) $T * \varphi \in C_{a p}$ for every $\varphi \in \mathcal{F}$.

(iii) There exist two functions $f$ and $g$ belonging to $C_{a p}$ and an ultradifferential operator $P$ of class $\left\{p !^{2}\right\}$ such that $T=P\left(D^{2}\right) f+g$.

(iv) The Gauss transform $u(x, t)$ of $T$ is almost periodic.

Proof. (i) $\Rightarrow$ (ii): Let $T$ be almost periodic. Then there exists a sequence $\left(P_{n}\right)$ of trigonometric polynomials converging to $T$ in $\mathcal{B}_{L^{\infty}}$. A simple computation yields that for every $\varphi \in \mathcal{F}, P_{n} * \varphi$ is also a trigonometric polynomial. It is easy to see that the set of translations $\left(\tau_{s} \varphi\right)_{s \in \mathbb{R}}$ is bounded in $\mathcal{A}_{L^{1}}$ where $\left(\tau_{s} \varphi\right)(t)=\varphi(t-s)$. Then it follows that

$$
\left(P_{n} * \varphi\right)(s)=\left\langle P_{n}, \tau_{s} \breve{\varphi}\right\rangle \longrightarrow\left\langle T, \tau_{s} \breve{\varphi}\right\rangle=(T * \varphi)(s)
$$

as $n \rightarrow \infty$ and the convergence is uniform on $\mathbb{R}$. Hence $T * \varphi \in C_{a p}$.

(ii) $\Rightarrow$ (iii): Let $v(t), w(t)$ and $P$ be as in Lemma 2.1. Define

$$
V(x, t)=\int_{0}^{\infty} E(x, t+s) v(s) d s, \quad W(x, t)=\int_{0}^{\infty} E(x, t+s) w(s) d s .
$$

Then $V(x, t)$ and $W(x, t)$ belong to $C^{\infty}\left(\mathbb{R}_{+}^{2}\right)$, and for fixed $t>0$ they also belong to $\mathcal{F}$ as functions of $x$.

By the assumption we have that for fixed $t>0$ the functions $T *_{x} V(x, t)$ and $T *_{x} W(x, t)$ belong to $C_{a p}$.

Also, we have

$$
\begin{aligned}
P(-d / d t) V(x, t) & =\int_{0}^{\infty} P(-d / d t) E(x, t+s) v(s) d s \\
& =\int_{0}^{\infty} P(-d / d s) E(x, t+s) v(s) d s \\
& =\int_{0}^{\infty} E(x, t+s) P(d / d s) v(s) d s \\
& =\int_{0}^{\infty} E(x, t+s)(-w(s)+\delta(s)) d s \\
& =-W(x, t)+E(x, t) .
\end{aligned}
$$

Therefore, we obtain the following:

$$
\begin{aligned}
P(-d / d t) V(x, t)+W(x, t) & =E(x, t), \\
P(-d / d t) T *_{x} V(x, t)+T *_{x} W(x, t) & =T *_{x} E(x, t), \\
P\left(D^{2}\right) T *_{x} V(x, t)+T *_{x} W(x, t) & =T *_{x} E(x, t) .
\end{aligned}
$$

Then by the uniqueness of the Cauchy problem for the heat equation in $[\mathrm{W}$ ] there exist functions $g(x)$ and $h(x)$ in $L^{\infty}$ such that

$$
T *_{x} V(x, t)=g(x) * E(x, t) \text { and } T *_{x} W(x, t)=h(x) * E(x, t) .
$$

In fact, since $T * V(x, 0)=g(x)$ and $T * W(x, 0)=h(x)$ it follows that $g(x), h(x) \in$ $C_{a p}$. 
(iii) $\Rightarrow$ (i): Let $T=P\left(D^{2}\right) g(x)+h(x)$ where $g(x), h(x) \in C_{a p}$. Note that every element in $C_{a p}$ is almost periodic in $\mathcal{B}_{L^{\infty}}$. So $P\left(D^{2}\right) g$ and $h$ are almost periodic in $\mathcal{B}_{L^{\infty}}$ by Lemma 3.4. Therefore, $T$ is almost periodic.

(ii) $\Rightarrow$ (iv): Since the heat kernel $E(x, t)$ belongs to the Sato space $\mathcal{F}$ for each $t>0$, the Gauss transform $u(x, t)=T * E(x, t)$ belongs to $C_{a p}$ for each $t>0$.

(iv) $\Rightarrow$ (ii): Assume that the Gauss transform

$$
u(x, t)=T_{y}(E(x-y, t))=\left(T * E_{t}\right)(x)
$$

of $T$ is almost periodic for each $t>0$. Then for every $\varphi \in \mathcal{F},\left(T * E_{t}\right) * \varphi=(T * \varphi) * E_{t}$ is an almost periodic function for each $t>0$, which converges uniformly to $T * \varphi$ as $t \rightarrow 0^{+}$, since $T * \varphi$ and $(T * \varphi)^{\prime}$ are bounded. Thus $T * \varphi$ is almost periodic for every $\varphi \in \mathcal{F}$.

As an application to the Dirichlet problem for the half plane for the case of hyperfunctions we state the following theorem without proof, which generalizes the result in [C2].

Theorem 3.6. Let $T \in \mathcal{B}_{L^{\infty}}$ be almost periodic. Then there exists a harmonic function $u(x, y)$ in the right half-plane such that

(i) for every $x>0$, the function $y \rightarrow u(x, y)$ is almost periodic;

(ii) $u(x, y) \rightarrow T$ in $\mathcal{B}_{L^{\infty}}$ as $x \rightarrow 0$.

\section{Almost periodic quasianalytic ultradistributions}

As mentioned in Section 1, Cioranescu has already obtained the characterization of almost periodic ultradistributions of Beurling type in [C2]. We give a similar result for quasianalytic ultradistributions of Roumieu type provided that $M_{p}$ satisfies the conditions (M.1), (M.2) and the following condition (C): There exists a positive integer $k$ such that $\liminf _{p \rightarrow \infty}\left(m_{k p} / m_{p}\right)^{2}>k$ where $m_{p}=M_{p} / M_{p-1} p=1,2, \cdots$.

The condition (C) is much weaker than (M.3). In fact, the condition (C) is equivalent to the fact that $M_{p}^{2}$ satisfies (M.3). Hereafter, we always assume the conditions (M.1), (M.2) and (C). See CK for more details.

Here we use the following Lemma which is a modified version of Lemma 2.1.

Lemma 4.1 ([CK]). For any $h>0$ and $\epsilon>0$ there exist functions $v(t) \in C_{c}^{\infty}([0, \epsilon])$, $w(t) \in C_{c}^{\infty}([\epsilon / 2, \epsilon])$ and an ultradifferential operator $P(d / d t)$ such that

$$
\begin{gathered}
\left|v^{(k)}(t)\right| \leq C h^{-k} M_{k}^{2}, \quad k=0,1, \cdots, \\
|v(t)| \leq C \exp \left(-M^{*}(h / t)\right), \quad 0<t<\infty, \\
P(d / d t)=\sum_{k=0}^{\infty} a_{k}(d / d t)^{k},\left|a_{k}\right| \leq C_{1} h_{1}^{k} / M_{k}^{2}, \quad 0<h_{1}<h, \\
P(d / d t) v(t)+w(t)=\delta,
\end{gathered}
$$

where $M^{*}(t)=\sup _{p} \log \frac{p ! t^{p}}{M_{p}^{2}}$.

Employing Lemma 4.1 and adapting the proof of Theorem 3.1 we can characterize bounded quasianalytic ultradistributions as follows. 
Lemma 4.2. The following statements are equivalent:

(i) $T \in \mathcal{D}^{\prime}{ }_{L^{\infty}}^{\left\{M_{p}\right\}}$.

(ii) $T * \varphi \in L^{\infty}$ for every $\varphi \in \mathcal{D}_{L^{1}}^{\left\{M_{p}\right\}}$.

(iii) There exist two functions $f, g \in C_{b}$ and an ultradifferential operator of class $\left\{M_{p}^{2}\right\}$ such that $T=P\left(D^{2}\right) f+g$.

(iv) The Gauss transform $u(x, t)$ of $T$ belongs to $C^{\infty}\left(\mathbb{R}_{+}^{2}\right)$ and satisfies the following:

$$
\left(\partial_{t}-\Delta\right) u(x, t)=0 \quad \text { in } \mathbb{R}_{+}^{2}
$$

for every $\epsilon>0$ there exists a constant $C>0$ such that

$$
\|u(x, t)\|_{L^{\infty}\left(\mathbb{R}_{x}\right)} \leq C \exp \left(M^{*}(\epsilon / t)\right) \quad \text { in } \mathbb{R}_{+}^{2},
$$

where $M^{*}(t)=\sup _{p} \log \frac{p ! t^{p}}{M_{p}^{2}}$; and $u(\cdot, t) \rightarrow T$ as $t \rightarrow 0^{+}$in the sense that

$$
T(\varphi)=\lim _{t \rightarrow 0^{+}} \int_{\mathbb{R}} u(x, t) \varphi(x) d x, \quad \varphi \in \mathcal{D}_{L^{1}}^{\left\{M_{p}\right\}} .
$$

Making use of Lemma 4.1 and Lemma 4.2 and suitably modifying the proof of Theorem 3.5 we obtain the following characterization of almost periodic ultradistributions which unify previous results on characterizations of various almost periodic generalized functions.

Theorem 4.3. For $T \in \mathcal{D}_{L^{\infty}}^{\prime\left\{M_{p}\right\}}$ the following are equivalent:

(i) $T$ is almost periodic.

(ii) $T * \varphi \in C_{a p}$ for every $\varphi \in \mathcal{D}_{L^{1}}^{\left\{M_{p}\right\}}$.

(iii) There exist two functions $f, g \in C_{a p}$ and an ultradifferential operator $P$ of class $\left\{M_{p}^{2}\right\}$ such that $T=P\left(D^{2}\right) f+g$.

(iv) The Gauss transform $u(x, t)$ of $T$ is almost periodic.

\section{ACKNOWLEDGEMENT}

We would like to express our sincere gratitude to the referee for careful reading and correction of our paper.

\section{REFERENCES}

[C1] I. Cioranescu, On the abstract Cauchy problem in spaces of almost periodic distributions, J. Math. Anal. Appl 148 (1990), 440-462. MR 91d:34065

[C2] The characterization of the almost periodic ultradistributions of Beurling type, Proc. Amer. Math. Soc 116 (1992), 127-134. MR 92k:46063

[CK] S.-Y. Chung and D. Kim, Representation of quasianalytic ultradistributions, Ark. Mat. 31 (1993), 51-60. MR 94e:46069

[CKL] S.-Y. Chung, D. Kim and E. G. Lee, Periodic hyperfunctions and Fourier series, Proc. Amer. Math. Soc. 128 (2000), 2421-2430. CMP 99:05

[H] L. Hörmander, The analysis of linear partial differential operators I, Springer-Verlag, Berlin-New York, 1983. MR 85g:35002a

[K] H. Komatsu, Ultradistributions. I, J. Fac. Sci. Univ. Tokyo. Sect. IA Math 20 (1973), 25-105. MR 47:9277

[KCK] K. H. Kim, S.-Y. Chung and D. Kim, Fourier hyperfunctions as the boundary values of smooth solutions of heat equations, Publ. RIMS, Kyoto Univ 29 (1993), 289-300. MR 94m:46076

[M] T. Matsuzawa, A calculus approach to hyperfunctions II, Trans. Amer. Math. Soc 313 (1990), 619-654. MR 90g:46062 
[S] L. Schwartz, Théorie des distributions, Hermann, Paris, 1966. MR 35:730

[W] D. V. Widder, The heat equation, Academic Press, New York, 1975. MR 57:6840

Department of Mathematics, Kunsan National University, Kunsan 573-360, Korea

E-mail address: jychung@ks.kunsan.ac.kr

Department of Mathematics, Sogang University, Seoul 121-742, Korea

E-mail address: sychung@ccs.sogang.ac.kr

Department of Mathematics, Seoul National University, Seoul 151-742, Korea

E-mail address: dhkim@math.snu.ac.kr

Department of Mathematics, Seoul National University, Seoul 151-742, Korea

E-mail address: ciel@math.snu.ac.kr 